\title{
Farming in the HERA-B Experiment
}

\author{
J.M.Hernández ${ }^{1}$ \\ for the SLT, L4 and DAQ groups of the HERA-B Experiment \\ ${ }^{1}$ Deutsches Elektronen-Synchrotron, DESY Zeuthen, Platanenallee 6, D-15738 Zeuthen (Germany)
}

\begin{abstract}
The HERA-B data acquisition and triggering systems make use of Linux PC farms for triggering and online event reconstruction. We present in this paper the requirements, implementation and performance of both PC farms. They have been fully working during the 2000 detector and trigger commissioning run.
\end{abstract}

\section{INTRODUCTION}

The HERA-B experiment [1] is a fixed target hadronic b-factory at HERA. It is dedicated to the measurement of CP violation in the decay of $\mathrm{B}$ mesons. The data acquisition and triggering system must cope with more than half a million detector channels, a $40 \mathrm{MHz}$ interaction rate and a signal to background ratio of around $10^{-10}$. A highly selective multi-level trigger with a suppression factor of $10^{-6}$, and a networked and high bandwidth data acquisition system, to handle the huge amount of data involved, have been implemented.

\section{THE HERA-B TRIGGER}

The $b \bar{b}$ production cross section in proton-nucleus interactions at the HERA-B center of mass energy $(\sqrt{s} \sim 40$ $\mathrm{GeV}$ ) is around $10^{-6}$ the cross section of inelastic interactions. To filter events coming from decays of B mesons, the trigger makes use of the fact that the $\mathrm{B}$ mesons have high mass and long lifetime and therefore the decay products should have high transverse momentum and should come from a detached vertex well separated from the primary interaction in the target. HERA-B is particularly interested in the reactions $B \rightarrow J / \Psi X$ with a $J / \Psi$ particle in the final state. The clear trigger signature is a pair of high $p_{T}$ leptons which form a secondary vertex and whose invariant mass is close to the $J / \Psi$ mass.

HERA-B implements a multi-level trigger to filter a few $\mathrm{Hz}$ of interesting events from a $40 \mathrm{MHz}$ interaction rate.

In figure II the first table shows for each of the trigger levels the input rate, the input data volume and the time available for the trigger decision. The second table contains the data flow into the different hardware components of the DAQ. The number of detector channels is 550000 and the uncompressed (compressed) event size is 500000 (120000) bytes respectively.

The large input event rate forces the first level trigger (FLT) to be a hardware trigger entirely build from specialized processors. The FLT combines lepton candidates from the muon detector and the electromagnetic calorimeter with hits in four of the tracking super-layers. It determines the momenta of the tracks by imposing a rough vertex constraint in the target and cuts on the invariant mass of pairs of candidates around the

\begin{tabular}{|c|c|c|c|}
\hline \multirow[t]{2}{*}{ Level } & \multirow{2}{*}{$\begin{array}{r}\text { Latency } \\
\text { sec }\end{array}$} & \multicolumn{2}{|c|}{ Input } \\
\hline & & $\begin{array}{r}\text { Trigger } \\
\mathrm{Hz}\end{array}$ & $\begin{array}{l}\text { Data } \\
\text { byte/s }\end{array}$ \\
\hline Pre & $10^{-6}$ & $10 \cdot 10^{6}$ & $90 \cdot 10^{9}$ \\
\hline 1 & $12 \cdot 10^{-6}$ & $10 \cdot 10^{6}$ & $10 \cdot 10^{9}$ \\
\hline 2 & $7 \cdot 10^{-3}$ & $50 \cdot 10^{3}$ & $250 \cdot 10^{6}$ \\
\hline 3 & $100 \cdot 10^{-3}$ & $500 \cdot 10^{0}$ & $250 \cdot 10^{6}$ \\
\hline 4 & $4 \cdot 10^{0}$ & $50 \cdot 10^{0}$ & $6 \cdot 10^{6}$ \\
\hline
\end{tabular}

\begin{tabular}{|l|r|}
\hline \multicolumn{2}{|c|}{ Data flow (bytes/s) } \\
\hline$\rightarrow$ L1 pipe & $5 \cdot 10^{12}$ \\
$\rightarrow$ L2 buffers & $25 \cdot 10^{9}$ \\
$\rightarrow$ L3 processors & $250 \cdot 10^{6}$ \\
$\rightarrow$ L4 processors & $6 \cdot 10^{6}$ \\
$\rightarrow$ tape & $2.4 \cdot 10^{6}$ \\
\hline
\end{tabular}

Figure 1: Input rate, data flow and latency of the trigger levels

$J / \Psi$ mass.

Due to the huge data volume and the small latency, the Second Level Trigger (SLT) code only processes from the events regions of interest (RoI) developed by the FLT. The complete event information is stored in a distributed system of Second Level buffers (SLB) waiting for the SLT trigger decision. The SLT is a software trigger running on a PC farm of 240 PCs. It refines the tracks found by the FLT adding more tracking super-layers and detector information. It also applies cuts on secondary vertices.

After passing the SLT, the events are built from the SLB's and processed at the same node using the Third Level Trigger code (TLT). The TLT looks at event properties beyond the triggered $J / \Psi$ tracks.

The events passing the TLT are sent to the online reconstruction Linux PC farm made up of additional 200 processors. The full event reconstruction is performed online to make most efficient use of computing resources and to minimize time delays between data taking and physics analysis. New calibration and alignment constants are derived online from the reconstructed events and if necessary fed back into the trigger processors to keep the performance of the trigger under detector variations.

\section{THE HERA-B DATA ACQUiSiTION SYSTEM}

Figure III depicts the HERA-B Data Acquisition System (DAQ) in connection with the different trigger levels. The HERA-B DAQ is a high bandwidth and high transaction rate system to serve the needs of the HERA-B high level triggers. The HERA-B high level triggers are software triggers running in two independent PC farms. 
The data path components in the DAQ are the following:

- ADSP2160 SHARC Processors: Digital Signal Processors used for data buffering in the SLB's and for the switching network connecting the SLB's and the Second Level Processors (SLP).

- Farm Processors: Intel Pentium PCs distributed in the SLT farm and the Online reconstruction farm.

- SHARC to PCI interface card [5] to connect the SLP's with the SHARC switch.

- Fast/Gigabit ethernet switches to connect the SLT farm with the Online reconstruction farm.

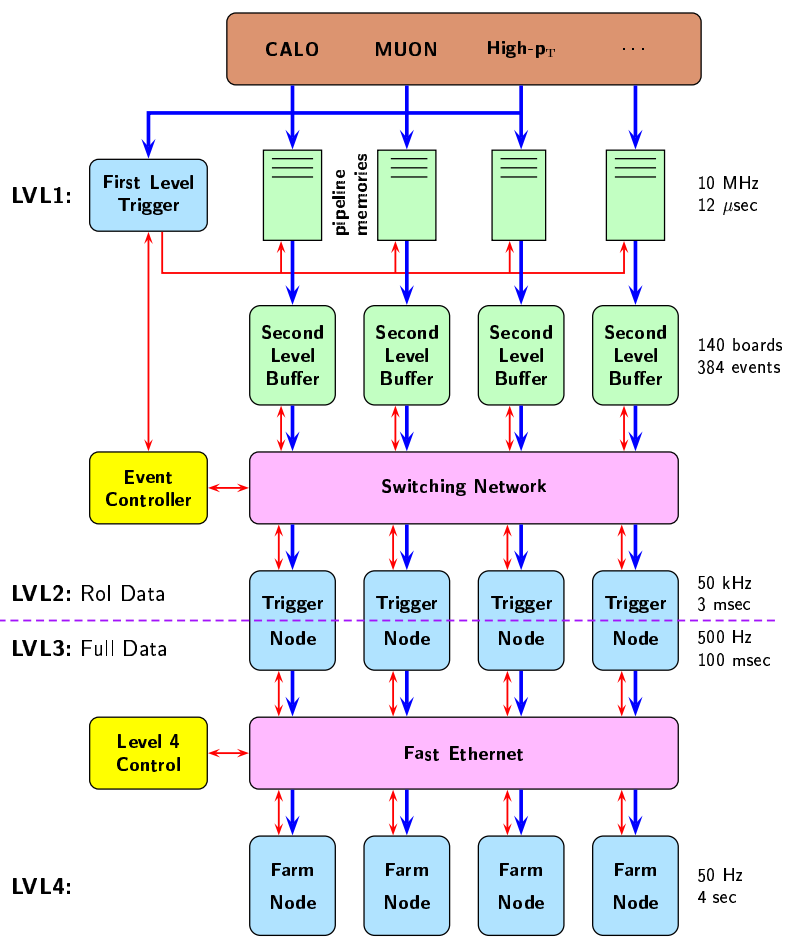

Figure 2: The HERA-B Data Acquisition and Trigger systems

The SLB's and SLP's are distributed in blocks (see figure III). Each SLB block is connected to all SLP blocks and vice versa via the SHARC switching network. To increase the maximum transaction rate in the sharc switch, there are two independent lines for the request of data from the SLP's to the SLB's and for the reply with the event data from the SLB's.

The SLT farm is connected to the Online reconstruction farm via a standard Fast(100Mbps)/Gigabit(1000Mbps) Ethernet switched network.

During the 2000 run, the performance reached in the communication SLB's $\Leftrightarrow$ SHARC SWITCH $\Leftrightarrow$ SLP's was a bandwidth of $1 \mathrm{Gbyte} / \mathrm{sec}$ and a maximum message rate of 2.6 MHz. This figures are above the design values of a required bandwidth of $500 \mathrm{Mbyte} / \mathrm{sec}(250 \mathrm{MB} / \mathrm{s}$ for the RoI traffic and $250 \mathrm{MB} / \mathrm{s}$ for the event building) and a maximum message rate of $1.8 \mathrm{MHz}$.
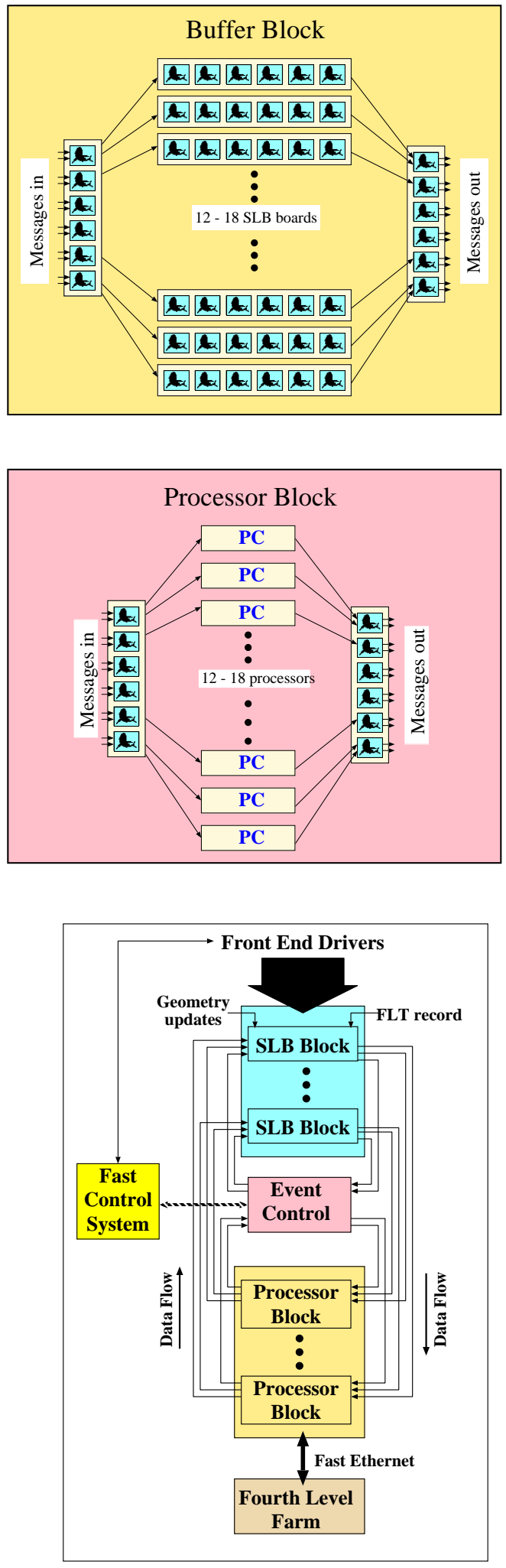

Figure 3: Topology of the connection SLB's $\Leftrightarrow$ SHARC SWITCH $\Leftrightarrow$ SLP's 
For more details on the HERA-B DAQ see in this proceedings [2]

\section{The SECond/Third LeVel Trigger PC FARM}

Given the already reduced output rate of $50 \mathrm{KHz}$ from the FLT, a PC FARM can be used to carry out the SLT algorithm [4]. The PCs have to have access to the detector data needed by the SLT algorithm. Each node takes care of processing a given event. Since the collection of the data of complete events at a rate of $50 \mathrm{KHz}$ would require an unattainable bandwidth in the data transfer from the Front-End-Electronics of the detector to the farm, the SLT algorithm must work on small regions (Regions of interest) in the events, typically $1 \%$ of the whole event size. The events are stored in the distributed system of second level buffers during the execution of the SLT algorithm.

\section{A. The SLT-SLB protocol}

As described in the previous section, the processors of the trigger farm are connected to the SLB's via a specialized network. The data transfer between the SLB's and the SLP's is managed by the Event Controller. The use of a standard switched network (e.g. ethernet) to allow the access of the data from all the detector channels with the required small latency and high bandwidth proved to be not possible at the time of the HERA-B DAQ design. Hence, a special network for this purpose based on digital signal processors of the type SHARC ADSP-21060 was designed and built. The SHARCs are also used for the SLB's and the Event Controller. For the connection between the SLP's and the SHARC switch, a interface card between the SHARC system and the PCI system bus of the PC's was specially developed for HERA-B .

The data transfer protocol between SLB's and SLP's managed by the event controller is as follows [3] (see figure A): As soon as the Event controller receives a new trigger accept from the FLT, the identifier of the event is communicated to one of the free SLP's. The Event Controller keeps a list of free SLP's and acts as well as buffer manager of the SLB event slots. The SLP first fetches from the corresponding SLB the FLT information of the event, which contains the track candidates. From this information, the data of the detector channels which lie in the Rol's defined by the tracks can be retrieved from the SLB's. For each step of the SLT, additional data is pulled from the SLB system.

If the event is accepted by the SLT, all the event data is retrieved from the SLB's and the event building is done. After the SLT decision, positive or not, the SLP informs the Event Controller that it is available for a new event. The Event controller informs the SLB's that the slot corresponding to the already processed event can be overwritten by a new coming event.

After the event building the same node executes the TLT algorithm. Using the same node for the SLT and TLT simplifies the protocol of the data transfer between SLB's and trigger processors.

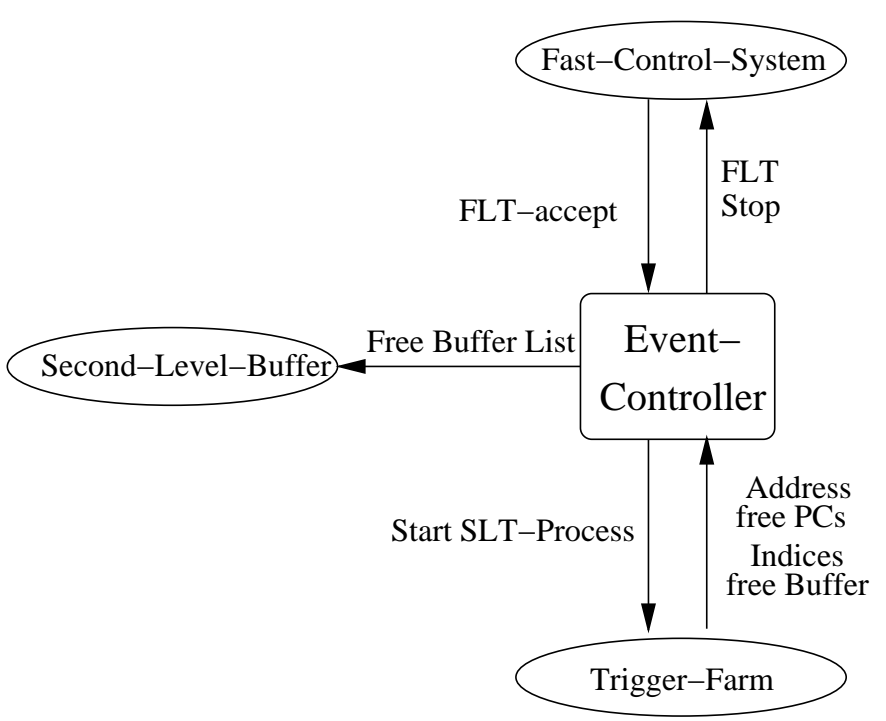

Figure 4: The SLB $\Leftrightarrow$ SLP data transfer protocol

The number of processors needed by the trigger farm is determined by the product of the input rate and the average latency of the trigger algorithm. For an average latency of around $3.8 \mathrm{msec}$ per event and an input rate of $50 \mathrm{KHz}, 190$ processors are needed for the SLT. For the TLT, given the input rate of $500 \mathrm{~Hz}$ and an average latency of $100 \mathrm{msec}, 50$ processors are needed. In total, the Second/Third Level trigger farm is made of 240 nodes.

The size of the storage in the SLB's has to be at least large enough to store the number of events which simultaneously are being processed in the SLT given by the number of nodes in the SLT farm. To cope with rate fluctuations in the FLT, more events should be stored. There is room for 250 to 350 events in the SLB system.

Unlike the pipeline storage system of the detector readout electronics in which the events are overwritten after a given period of time, in the SLB system every single event can in principle stay arbitrarily long. Therefore, the maximum latency for a given event in the SLT is not limited by the storage size of the SLB but only the average latency. This way the system can cope with fluctuations in the latency of the SLT algorithm which depends heavily on the number of candidates found by the FLT.

To avoid the overflow of the SLB's, the event controller sends a veto to the FLT when there is no room for more events in the SLB's. The veto is removed when a sufficient number of events have been processed by the SLT.

Since the SLP's have access to the whole data of a given event, the SLT algorithm can in principle make use of the information of regions of the events arbitrarily large. The only limitation is the bandwidth and the maximum transaction rate between SLB's and SLP's. The size of the RoI's can be changed at any time. In the same way, additional sub-detectors or detector channels outside the Rol's can be used. 


\section{B. The SLT/TLT Farm Hardware}

The SLT/TLT nodes are standard PCs equipped with offthe-shelf and custom made components. Among the standard components are the Intel Pentium III $400 \mathrm{MHz}$ processor, 64 MB SDRAM memory, floppy drive, a cheap graphics card and a $100 \mathrm{Mbit} / \mathrm{s}$ Fast Ethernet adapter. Specially developed for the connection to the SLB system was the SHARC to PCI interface adapter together with the driver code. The non-standard I/O driver copies data directly between the SHARC link interface and the user space. The throughput of the sharc-to-pci interface is $40 \mathrm{MB} / \mathrm{s}$ in both directions. Another remarkable feature of this link is the very small latency, around $1 \mu \mathrm{sec}$. There is another special interface card in the nodes for slow control using the CAN protocol. This serial line is used for powering up/down and reseting the nodes as well as for measuring temperatures and voltages in the mother board and the fan speed.

The nodes are disk-less. A reduced linux operating system is installed in a floppy disk from which they boot. At booting time the nodes issue a bootp request to the farm server to have an IP address assigned. The nodes NFS-mount a file-system from the server to have access to the executables of the trigger code. Only essential processes run in the nodes to avoid as much as possible process scheduling by the operating system. The trigger process should stay active as much as possible. No real time extensions of linux were necessary to ensure a good performance of the trigger code.

\section{Data transfer to the Online Reconstruction Farm}

The data transfer from the SLT/TLT farm to the online reconstruction farm is managed by a controller process running in one of the nodes of the SLT/TLT farm (see figure C). This controller keeps internally a list of free online reconstruction nodes. When a SLT/TLT node is ready to send one event to the reconstruction farm it requests via the sharc link from the controller the address (IP number and port) of one free online reconstruction node. The events are sent via a standard Fast Ethernet (100 Mbit/s) network with Gigabit Ethernet (1000 $\mathrm{Mbit} / \mathrm{s})$ uplinks.

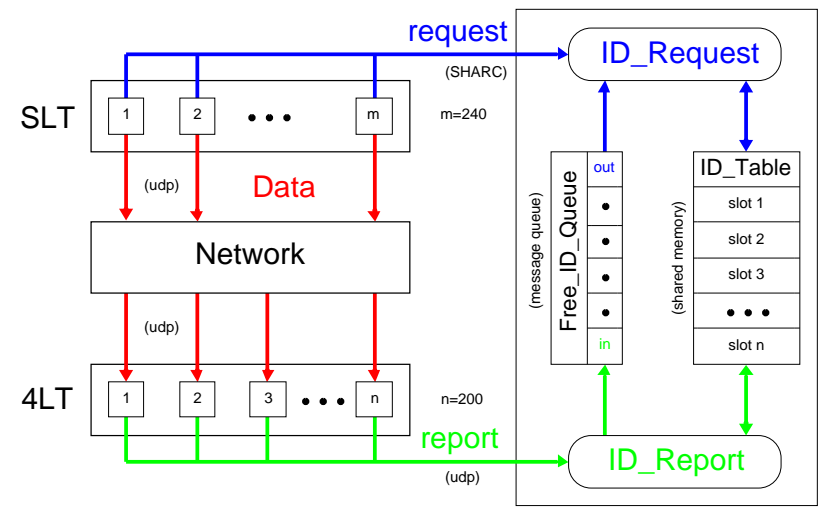

Figure 5: The TLT $\Leftrightarrow$ 4LT data transfer protocol

\section{The Fourth Level Online RECONSTRUCTION PC FARM}

Due to the vast amount of event data (around $20 \mathrm{~TB} / \mathrm{year}$ ) and the large event reconstruction time (around 4 sec/event), immediate data analysis can only be ensured by performing event reconstruction online before event data are archived. In addition event classification and event selection to further reduce the output rate from $50 \mathrm{~Hz}$ to $20 \mathrm{~Hz}$ are done in the fourth level farm (4LT) [6]. The SLT/TLT farm is a trigger system dominated by bandwidth and latency whereas the 4LT farm is dominated by processing power needs.

The main tasks of the 4LT system are:

- Full online event reconstruction,

- event classification,

- final event selection (4LT trigger step),

- data logging,

- data quality monitoring,

- preparation of data for online calibration and alignment,

- event re-processing during shutdown periods.

The system must guarantee event data transfer. Therefore a push architecture is used for the event data stream, whereas monitoring information is collected from the nodes by a pull architecture.

The requirements of the system can be summarized as follows: It needs to provide sufficient processing power and bandwidth, be flexible to handle widely spread processing times and data rates, be scalable to easily increase the processing power and stay within cost limits.

For a farm architecture, the following building blocks can be identified: processing nodes, data links, switching network, event flow control, file and data server.

To process events at a rate of $50 \mathrm{~Hz}$ with processing times of $4 \mathrm{sec}$ on a modern CPU (Intel Pentium III $500 \mathrm{MHz}$ ), 200 farm nodes are needed. The network must be capable of routing $5 \mathrm{MB} / \mathrm{sec}$ to the farm nodes and a similar amount of data from the system to archive.

\section{A. The 4LT Farm Hardware}

The concept and architecture of the 4LT farm allow to use off-the-shelf components. The processing power can be provided by modern PC-CPUs. The moderate bandwidth requirements can be met by Fast Ethernet.

As farm nodes, Intel CPUs were chosen which are housed in dual CPU PCs. The PCs are equipped with Intel Pentium III $500 \mathrm{MHz}$ processors, $256 \mathrm{MB}$ SDRAM and 13 GB EIDE disks. Each node can buffer of the order of 10 events in its local memory.

The network is built of 24-port CISCO Fast Ethernet switches (see figure A). In addition to the data stream which 
goes from TLT nodes to 4LT nodes and after processing to a central logger, control messages and monitoring data must be routed through the system. The farm nodes are grouped into eight so called mini-farms. The total bandwidth is divided accordingly. The backbones of the mini-farms are based on switches to ensure full bandwidth to/from the nodes concurrently. All mini-farms are connected to a central switch. Event data appear twice in the central switch, when being routed from TLT to 4LT and when being sent to the central logger. The data link to mass storage media is realized by means of Gigabit Ethernet. Event data is stored intermediately on large disks in the logger machine before being copied to tape. The full data stream leads to $20 \mathrm{~TB} /$ year which cannot be stored on disk. a small fraction of events $(\sim 1 \%)$ will be selected for direct access and provided to the user on disks.

One server PC provides NFS service for executables, and NIS services to the nodes of the farm.

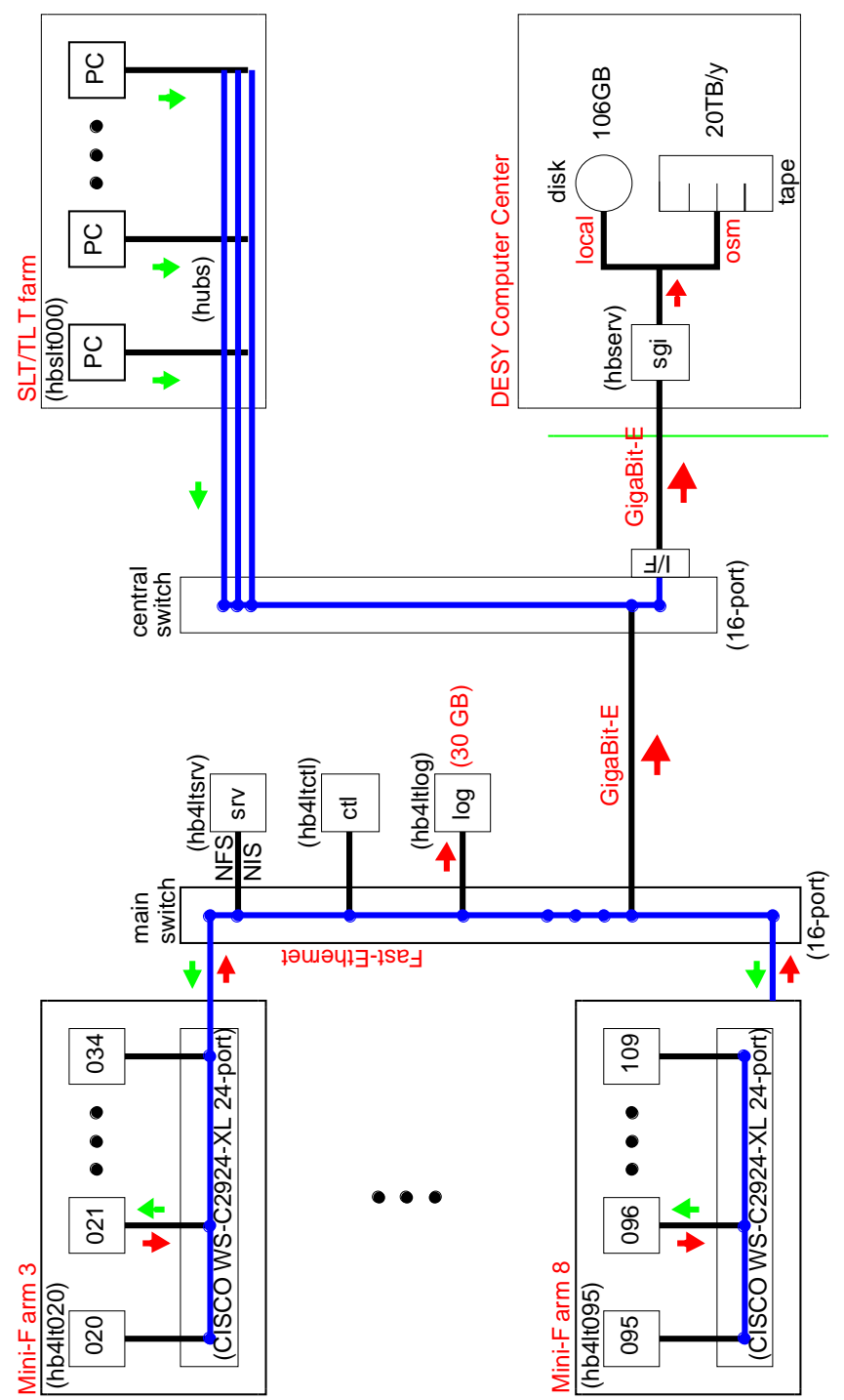

Figure 6: The ethernet network in the farms

\section{B. The 4LT Farm Software}

Figure B shows the process scheme of a 4LT farm node. Major tasks are housed in separate processes, using the HERAB message passing system between nodes and Unix standard interprocess communication within nodes.

Reconstruction, event classification and selection packages are contained in a frame program called ARTE. It provides memory management for event data stored in tables. ARTE is also used for offline analysis, including Monte Carlo event generation and detector simulation. ARTE contains interfaces to event data files for offline purposes as well as to shared memory segments for online usage on the 4LT farm. In the latter case, event data are received by a dedicated process from a TLT node initiated by the 4LT farm control. Output is done in a similar way. Event data are sent to the central logger in the computer center.

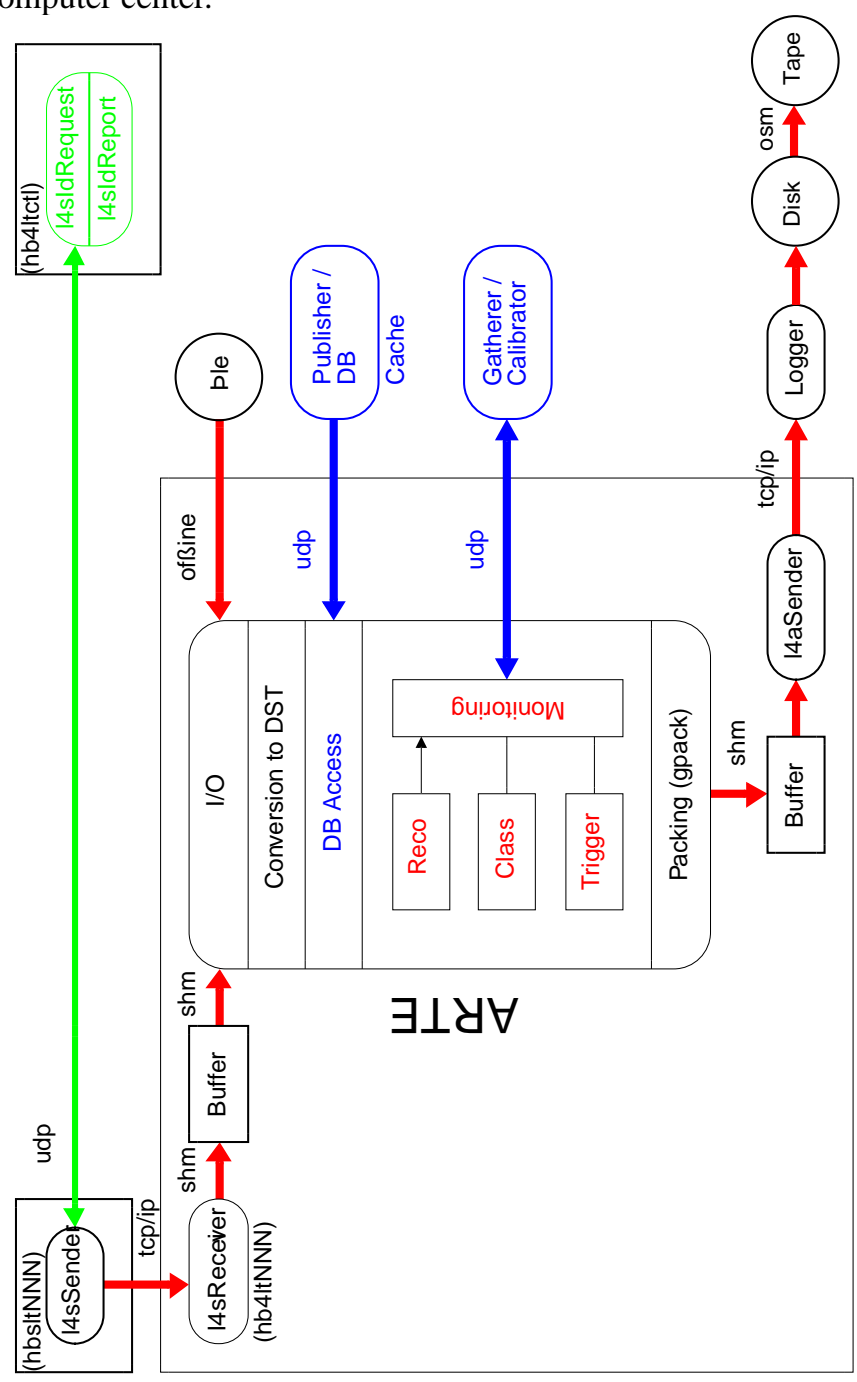

Figure 7: Processes in a 4LT node

\section{Online Calibration and Alignment}

During the reconstruction procedure, data which are needed to align and calibrate the detector are derived. To make use of the large statistics of up to 200 nodes providing such data in 
parallel, a scheme was developed to collect data in a central place (gatherer). Gathered data are then used to compute alignment and calibration constants which are if needed updated in the central database (see figure C).

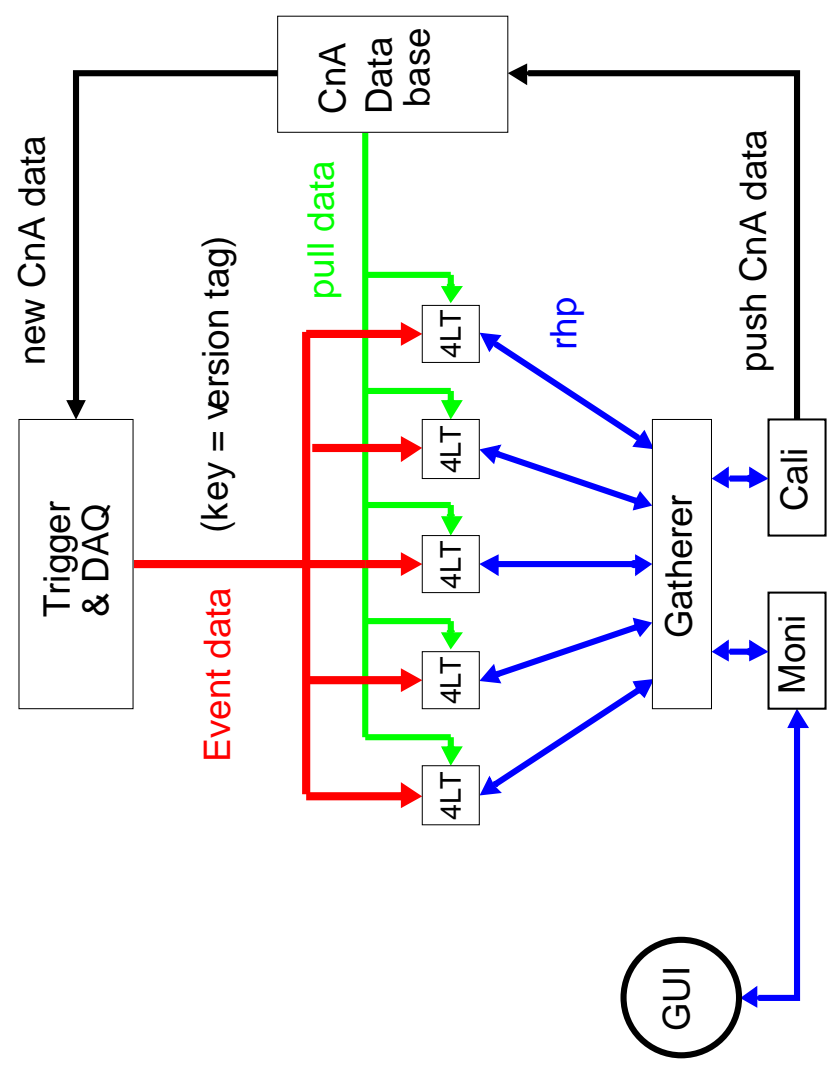

Figure 8: The ethernet network in the farms

In Hera-B all trigger levels and the online reconstruction depend on and have to access the most recent Calibration and Alignment (CnA) data. Updated CnA constants are produced online not only in the reconstruction farm but also in dedicated second level nodes. Those dedicated CnA nodes get calibration triggers from the DAQ at a sufficient rate.

The set of CnA constants used during data-taking at any time is identified by the so-called CnA key. This key is written in the event header. It allows an event to be associated with all the calibration and alignment data used in its trigger process and online reconstruction.

The format of the CnA constants as stored in the database servers might be different to the format required by the trigger processes. To centralize the formatting of the constants and save $\mathrm{CPU}$ processing time in the trigger processes, the so-called $\mathrm{CnA}$ formatters have been introduced in the system.

A pushed architecture has been designed for a low latency distribution of the CNA data to the Second level trigger processors. The updated CNA constants are multicasted using the fast and reliable SHARC links. On the other hand, the larger processing time of the online reconstruction nodes ( secs), allows a slower distribution using a pull architecture.
The reconstruction nodes fetch the updated CNA data from fast Database memory caches via fast ethernet.

The figure $\mathrm{C}$ illustrates the online distribution of $\mathrm{CnA}$ data to the trigger and online reconstruction processes.

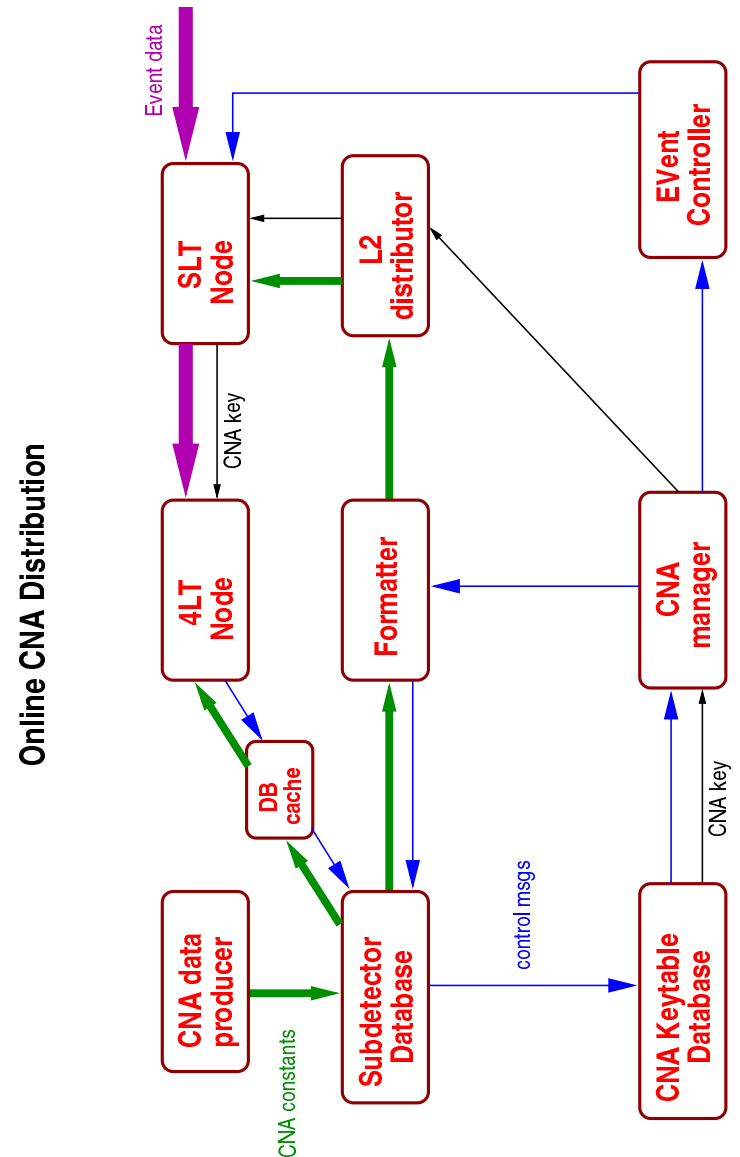

Figure 9: Online distribution of $\mathrm{CnA}$ constants

The distribution is initiated by the storage of new constants into active database servers which propagate the updates to the key server.The update is sent in turn to the CnA manager, the process in charge of the synchronization of the distribution. The CnA manager requests the Event Controller to pause the run and the involved formatters to build and distribute the updated constants to the trigger processes. Once the distribution is concluded successfully the Event Controller is contacted again to resume the run. The online reconstruction processes retrieve the new constants from fast memory database caches when events with the new key arrive to the farm.

During the 2000 run both the loading of the CnA constants at booting time in the trigger and reconstruction processes and the online distribution of updated constants were done routinely. The attained performance of the loading of the constants in the second level processes through the SHARC switch was $1 \mathrm{~GB} / \mathrm{sec}$. With the introduction of fast memory database caches, the performance of the loading of the constants in the online reconstruction farm was found to be sufficient, of the order of $15 \mathrm{MB} / \mathrm{sec}$. 


\section{Event data Re-processing}

A system to exploit the CPU power of the 4LT farm for event data re-processing has been setup. It works similarly to the usual online processing scheme and makes use of most of the online processes, in particular the logging and archiving facilities. Only the data source is different. Instead of passing raw event data from the DAQ system (via the SLT/TLT farm) to the 4LT farm nodes, a process retrieves data files from tape and distributes event by event to the 4LT farm nodes. The system proved to be very useful and robust. As the detector is better understood, the reconstruction packages further developed and improved CnA constants are produced, the event data need to be reprocessed. The performance achieved was an event reprocessing rate of $30 \mathrm{~Hz}$ only limited by the CPU power of the logging machine.

\section{SUMMARY}

HERA-B has successfully implemented trigger and online reconstruction farms in the DAQ. A low latency and high bandwidth switch for the Second Level Trigger system based on SHARC Digital Signal Processors has been built. The SLT/TLT trigger and the online reconstruction farms are connected via a standard Fast/Gigabit Ethernet switched network. The trigger farm allows the implementation of flexible second and third level trigger codes. The online reconstruction farm allows immediate data analysis, high quality data quality monitoring and online calibration and alignment.
Update $\mathrm{CnA}$ constants are fed back online into the trigger and online reconstruction farms. A system has been setup to exploit the CPU power of the farms to carry out data re-processing during shutdown periods.

PC farms running Linux provide a flexible, scalable and low cost solution for triggering in HEP experiments.

\section{REFERENCES}

[1] HERA-B Collaboration. "HERA-B, and experiment to study $\mathrm{CP}$ violation in the $\mathrm{B}$ system using and internal target at the HERA proton ring", DESY-PRC 95/01

[2] J.M.Hernández, D.Ressing, V.Rybnikov, F.Sánchez, G.Wagner. "HERA-B Data Acquisition System", These proceedings.

[3] G.Wagner. "Aufbau und Test der mit Digitalen Signal Prozessoren realisierten Komponenten des Datennahmesystem von HERA-B.”. Ph.D.Dissertation. Universitaet Hamburg, 2000

[4] M.Dam. "Second and Third Level Trigger Systems for the HERA-B experiment." Proceedings of the CHEP 98 conference Chicago, September 1998.

[5] K.H.Sulanke and I.C.Legrand. "Fast PCI Communication Interfaces for Online Distributed Processing Systems." Proceedings of the CHEP 98 conference Chicago, September 1998.

[6] A.Gellrich et al. "The Fourth Level Trigger Online Reconstruction Farm for HERA-B." Proceedings of the CHEP 98 conference Chicago, September 1998. 